\title{
A New Hyperbolic Area Formula of a Hyperbolic Triangle and Its Applications
}

\author{
Hui Bao and Xingdi Chen \\ Department of Mathematics, Huaqiao University, Quanzhou, Fujian 362021, China \\ Correspondence should be addressed to Xingdi Chen; chxtt@hqu.edu.cn
}

Received 14 May 2014; Accepted 25 July 2014; Published 13 August 2014

Academic Editor: Ming-Sheng Liu

Copyright ( $) 2014 \mathrm{H}$. Bao and X. Chen. This is an open access article distributed under the Creative Commons Attribution License, which permits unrestricted use, distribution, and reproduction in any medium, provided the original work is properly cited.

\begin{abstract}
We study some characterizations of hyperbolic geometry in the Poincaré disk. We first obtain the hyperbolic area and length formula of Euclidean disk and a circle represented by its Euclidean center and radius. Replacing interior angles with vertices coordinates, we also obtain a new hyperbolic area formula of a hyperbolic triangle. As its application, we give the hyperbolic area of a Lambert quadrilateral and some geometric characterizations of Lambert quadrilaterals and Saccheri quadrilaterals.
\end{abstract}

\section{Introduction}

Hyperbolic geometry was created in the first half of the nineteenth century in order to prove the dependence of Euclid's fifth postulate on the first four ones. It was extensively studied by Nikolai Lobachevskii and Johann Bolyai. Because of this, hyperbolic geometry is also known as BolyaiLobachevskian geometry. The basis necessary for an analytic study of hyperbolic geometry was laid by Leonhard Euler, Gaspard Monge, and Friedrich Gauss in their investigation of curved surfaces. Later, two of the most famous analytic models of the hyperbolic geometry were built, which are known as the Klein model and the Poincaré model in the name of their inventors [1]. One can refer to [2-4] for more history about hyperbolic geometry.

For a complex number $z$, we assume that $z=x+i y$, where $x, y \in \mathbf{R}$. For convenience, we denote by $z_{\mathrm{A}}=x_{\mathrm{A}}+i y_{\mathrm{A}}$ the coordinate of the point $A$. We denote the complex plane, the unit disk, and the upper half plane by $\mathbf{R}^{2}, \mathbf{B}^{2}$, and $\mathbf{H}^{2}$, respectively. We denote by $S^{1}(c, r)$ the Euclidean circle with center $c$ and radius $r$. Let $J[a, b]$ be the hyperbolic geodesic segment joining $a$ and $b$ when $a, b \in \mathbf{B}^{2}$ or $\mathbf{H}^{2}$, or the hyperbolic geodesic ray with one of the two points $a$ and $b$ is on $\partial \mathbf{B}^{2}$ or $\partial \mathbf{H}^{2}$. Let $\Omega \subset \mathbf{R}^{2}$ be a simply connected domain of hyperbolic type and let $\rho_{\Omega}(z)|d z|$ be the hyperbolic metric of $\Omega$ with the Gaussian curvature -1 . In particular, the hyperbolic metric density in $\mathbf{H}^{2}$ and $\mathbf{B}^{2}$ with the Gaussian curvature -1 is given by

$$
w_{\mathrm{H}^{2}}=\frac{1}{y}, \quad w_{\mathrm{B}^{2}}=\frac{2}{1-|z|^{2}},
$$

respectively.

Define the hyperbolic area of a measurable subset $E$ of $\Omega$ as

$$
A_{\text {hyp }}(E)=\iint_{E} \rho_{\Omega}(z)^{2}|d z|^{2} .
$$

Particularly, if $E$ is a measurable subset in $\mathbf{B}^{2}$, then

$$
A_{\text {hyp }}(E)=\iint_{E}\left(\frac{2}{1-|z|^{2}}\right)^{2} d x d y .
$$

The integrand at (3) is replaced with $1 / y^{2}$ when $E \subseteq \mathbf{H}^{2}$. We also denote the Euclidean area of a measurable subset $E \subset \Omega$ by $A_{\text {euc }}(E)$. For any rectifiable curve $\gamma$ in $\Omega$, the hyperbolic length of $\gamma$ is

$$
l_{\text {hyp }}(\gamma)=\int_{\gamma} \rho_{\Omega}(z)|d z| .
$$


If $\Omega$ is $\mathbf{B}^{2}$ or $\mathbf{H}^{2}$, then the integrands in (4) are $2 /\left(1-|z|^{2}\right)$ and $1 / y$, respectively. The hyperbolic distance between two points $z_{1}, z_{2} \in \Omega$ is defined by

$$
d_{\text {hyp }}\left(z_{1}, z_{2}\right)=\inf _{\gamma} l_{\text {hyp }}(\gamma) \text {, }
$$

where the infimum is taken over all rectifiable curves in $\Omega$ joining $z_{1}$ and $z_{2}$. We say that a curve $\gamma:[0,1] \rightarrow \Omega$ is a hyperbolic geodesic joining $\gamma(0)$ and $\gamma(1)$ if, for all $t \in(0,1)$, it follows

$$
d_{\text {hyp }}(\gamma(0), \gamma(1))=d_{\text {hyp }}(\gamma(0), \gamma(t))+d_{\text {hyp }}(\gamma(t), \gamma(1)) \text {. }
$$

If there exists a polygon enclosed by $n$ hyperbolic geodesics, then we call it a hyperbolic $n$ polygon. Particularly, if $n$ takes 3 or 4 , then we call it a hyperbolic triangle and hyperbolic quadrilateral, respectively. The interior angle of a hyperbolic polygon denotes the intersectional angle of the tangents of two geodesic arcs at the vertex. If there exists a hyperbolic quadrilateral with angles $(\pi / 2, \pi / 2, \phi, \pi / 2), 0 \leq$ $\phi<\pi / 2$, then it is said to be a Lambert quadrilateral [5, p. 156]. If there exists a hyperbolic quadrilateral with angles $(\pi / 2, \pi / 2, \phi, \phi), 0 \leq \phi<\pi / 2$, then it is called a Saccheri quadrilateral [5, p. 156].

Given two nonempty subsets $A, B$ of $G$, let $d_{\rho}(A, B)$ denote the hyperbolic distance between them, defined as

$$
d_{\rho}(A, B)=\inf _{z_{1} \in A, z_{2} \in B} \rho_{G}\left(z_{1}, z_{2}\right)
$$

where $\rho_{G}\left(z_{1}, z_{2}\right)$ stands for the hyperbolic distance between two points $z_{1}$ and $z_{2}$.

We also need the following three explicit formulas:

$$
\begin{gathered}
\cosh \rho_{\mathbf{H}^{2}}\left(z_{1}, z_{2}\right)=1+\frac{\left|z_{1}-z_{2}\right|^{2}}{2 y_{1} y_{2}} \\
\rho_{\mathbf{B}^{2}}\left(z_{1}, z_{2}\right)=2 \operatorname{arth} \frac{\left|z_{1}-z_{2}\right|}{\left.\sqrt{\left|z_{1}-z_{2}\right|^{2}+\left(1-\left|z_{1}\right|^{2}\right)\left(1-\left|z_{2}\right|^{2}\right.}\right)}
\end{gathered}
$$

for all $z_{1}, z_{2} \in \mathbf{B}^{2}$. In particular, for $z \in \mathbf{B}^{2}$,

$$
\rho_{\mathbf{B}^{2}}(0, z)=\log \frac{1+|z|}{1-|z|}=2 \operatorname{arth}|z| .
$$

The above basic facts can be found in our standard references $[5,6]$ and in many other sources on hyperbolic geometry such as $[7,8]$. Far-reaching and specialized advanced texts discussing hyperbolic geometry include [9, 10].

Hyperbolic triangles and Lambert quadrilaterals are fundamental geometric quantities for the study of hyperbolic geometry theory and its applications. Curien and Werner [11] constructed random triangulations of the Poincaré disc by hyperbolic triangles. Demirel [12], Yang and Fang [13, 14] gave characterizations of Möbius transformations by use of hyperbolic triangles or Lambert quadrilaterals. Pambuccian
[15] showed that mappings preserving the area equality of hyperbolic triangles are motions. One can see [16-18] for more characterizations about quasiconformal mappings and harmonic quasiconformal mappings in the sense of hyperbolic metrics.

Further study of geometric properties of hyperbolic triangles, Lambert quadrilaterals, and hyperbolic polygons also raises one's interest. Rostamzadeh and Taherian [19] considered the Klein model of the real hyperbolic plane and gave a new definition of its defect and area. A hyperbolic area formula and the radius of the inscribed circle of a hyperbolic triangle in the Poincaré model can be found in [5, p. 150, 152]. Recently, Vuorinen and Wang [20] obtained sharp bounds for the product and the sum of two hyperbolic distances between two opposite sides of hyperbolic Lambert quadrilaterals in the unit disk. Kanesaka and Nakamura [21] gave an explicit hyperbolic area formula for a $\theta$-acute triangle. One can refer to [22-24] for distortion estimates of hyperbolic areas of measurable subsets under quasiconformal mappings.

By the hyperbolic radius of a disc, the following is shown.

Theorem A (see [5]). (1) The area of a hyperbolic disc of radius $r$ is $4 \pi \sinh ^{2}(r / 2)$.

(2) The length of a hyperbolic circle of radius $r$ is $2 \pi \sinh r$.

Instead of a hyperbolic radius, we use the Euclidean center and radius of Euclidean disc in $\mathbf{H}^{2}$ to give its hyperbolic area formula as follows.

Theorem 1. For Euclidean disc $D_{1}=\left\{z:|z-(a+b i)|<R_{1}\right\} \subseteq$ $\mathbf{H}^{2}$, the hyperbolic area of $D_{1}$ is

$$
\begin{gathered}
A_{\text {hyp }}\left(D_{1}\right)=2 \pi\left(\frac{b}{\sqrt{b^{2}-R_{1}^{2}}}-1\right), \\
l_{\text {hyp }}\left(\partial D_{1}\right)=\frac{2 \pi R_{1}}{\sqrt{b^{2}-R_{1}^{2}}},
\end{gathered}
$$

where $b>R_{1}>0$.

Area formula for a hyperbolic polygon can be determined by its interior angles [5, p. 150].

Theorem B. For any hyperbolic triangle $T(A, B, C)$ with interior angles $\alpha, \beta, \gamma$,

$$
A_{\text {hyp }}(T(A, B, C))=\pi-(\alpha+\beta+\gamma) \text {. }
$$

In this paper, instead of interior angles, we will use the coordinates of vertexes of a hyperbolic triangle to give another hyperbolic area formula. We first study a hyperbolic triangle with a vertex at infinity.

Theorem 2. Let $z_{1}, z_{2} \in \mathbf{H}^{2}$ with $z_{1}=x_{1}+i y_{1}, z_{2}=x_{2}+$ $i y_{2}\left(x_{1} \neq x_{2}\right)$. 
(1) If $\left(x_{1}-c\right)\left(x_{2}-c\right)>0$,

$$
A_{\text {hyp }}\left(T\left(z_{1}, z_{2}, \infty\right)\right)=\left|\arctan \frac{y_{1}}{\left|c-x_{1}\right|}-\arctan \frac{y_{2}}{\left|c-x_{2}\right|}\right| .
$$

(2) If $\left(x_{1}-c\right)\left(x_{2}-c\right)<0$,

$$
\begin{aligned}
A_{\text {hyp }} & \left(T\left(z_{1}, z_{2}, \infty\right)\right) \\
= & \pi-\left|\arctan \frac{y_{1}}{\left|c-x_{1}\right|}\right|-\left|\arctan \frac{y_{2}}{\left|c-x_{2}\right|}\right|,
\end{aligned}
$$

where $c=\left(\left|z_{1}\right|^{2}-\left|z_{2}\right|^{2}\right) / 2\left(x_{1}-x_{2}\right)$.

For a general hyperbolic triangle in $\mathbf{H}^{2}$, its hyperbolic area can be represented by four hyperbolic triangles with a vertex at infinity (see Remark 6).

As some applications of the hyperbolic area formula of a hyperbolic triangle, we first give an explicit formula for the hyperbolic area of a Lambert quadrilateral in Section 3. Moreover, we obtain the length of two hyperbolic diagonal lines in a Lambert quadrilateral in $\mathbf{B}^{2}$ as follows.

Theorem 3. Let $\mathrm{Q}\left(v_{a}, v_{b}, v_{c}, v_{d}\right)$ be the Lambert quadrilateral in $\mathbf{B}^{2}$ with $v_{c}=t e^{i \theta}, 0<\theta<\pi / 2$. Then

$$
\begin{gathered}
\rho_{\mathrm{B}^{2}}\left(v_{a}, v_{c}\right)=\operatorname{arth} \frac{2 t}{1+t^{2}}, \\
\rho_{\mathbf{B}^{2}}\left(v_{b}, v_{d}\right)=2 \operatorname{arth} \sqrt{\frac{t_{1}^{2}+t_{2}^{2}}{1+t_{1}^{2} t_{2}^{2}}},
\end{gathered}
$$

where $t_{1}=\left|v_{b}-v_{a}\right|, t_{2}=\left|v_{d}-v_{a}\right|$.

Some geometric characterizations of Saccheri quadrilaterals are studied and a relation about two hyperbolic distances between two pairs of opposite sides is given in Section 4 (see Theorem 14).

\section{Hyperbolic Areas of Hyperbolic Triangles in $\mathbf{H}^{2}$}

Proof of Theorem 1. Let $D_{2}=\left\{z:|z|<R_{2}\right\}$ be Euclidean disc in $\mathbf{B}^{2}$. By direct calculation, the hyperbolic area of $D_{2}$ is given by

$$
\begin{aligned}
A_{\text {hyp }}\left(D_{2}\right) & =\iint_{D_{2}}\left(\frac{2}{1-|z|^{2}}\right)^{2} d x d y \\
& =\int_{0}^{2 \pi} \int_{0}^{R_{2}} r\left(\frac{2}{1-r^{2}}\right)^{2} d r d \theta=\frac{4 \pi R_{2}^{2}}{1-R_{2}^{2}}
\end{aligned}
$$

Let $f(z)=f_{2} \circ f_{1}(z)$, which maps $D_{1}=\{z: \mid z-(a+$ $\left.b i) \mid<R_{1}\right\} \subseteq \mathbf{H}^{2}$ onto the disk with center 0 and radius $\left(b-\sqrt{b^{2}-R_{1}^{2}}\right) / R_{1}$, where

$$
\begin{gathered}
f_{1}(z)=\frac{z-(a+2 b i)}{z-(a-2 b i)}, \quad f_{2}(z)=t \cdot \frac{z-s}{z-t}, \\
s=1-\frac{4 b\left(2 b-\sqrt{b^{2}-R_{1}^{2}}\right)}{3 b^{2}+R_{1}^{2}}, \\
t=1-\frac{4 b\left(2 b+\sqrt{b^{2}-R_{1}^{2}}\right)}{3 b^{2}+R_{1}^{2}} .
\end{gathered}
$$

Then we have

$$
\begin{gathered}
A_{\text {hyp }}\left(D_{1}\right)=2 \pi\left(\frac{b}{\sqrt{b^{2}-R_{1}^{2}}}-1\right), \\
l_{\text {hyp }}\left(\partial D_{1}\right)=\frac{2 \pi R_{1}}{\sqrt{b^{2}-R_{1}^{2}}} .
\end{gathered}
$$

Lemma 4. Let $\gamma$ be a hyperbolic geodesic in $\mathbf{H}^{2}$ with two endpoints $A=i t, B=t e^{i \theta}(0 \leq \theta \leq \pi, \theta \neq \pi / 2, t>0)$. Then the hyperbolic area of the hyperbolic triangle $T(A, B, \infty)$ is given as follows:

$$
A_{\text {hyp }}(T(A, B, \infty))=\left|\theta-\frac{\pi}{2}\right| .
$$

Proof.

Case 1. If $0 \leq \theta<\pi / 2$, then

$$
\begin{aligned}
A_{\text {hyp }}(T(A, B, \infty)) \\
\quad=\iint_{T(A, B, \infty)} \frac{1}{y^{2}} d x d y=\int_{0}^{t \cos \theta} d x \int_{\sqrt{t^{2}-x^{2}}}^{\infty} \frac{1}{y^{2}} d y \\
=\arcsin (\cos \theta)=\arcsin \left(\sin \left(\frac{\pi}{2}-\theta\right)\right) \\
\quad=\frac{\pi}{2}-\theta .
\end{aligned}
$$

Particularly, when $\theta=0$, then $A_{\text {hyp }}(T(A, B, \infty))=\pi / 2$.

Case 2. If $\pi / 2<\theta \leq \pi$, we have

$$
\begin{aligned}
A_{\text {hyp }}(T(A, B, \infty)) \\
\quad=\iint_{T(A, B, \infty)} \frac{1}{y^{2}} d x d y=\int_{t \cos \theta}^{0} d x \int_{\sqrt{t^{2}-x^{2}}}^{\infty} \frac{1}{y^{2}} d y \\
\quad=-\arcsin (\cos \theta)=-\arcsin \left(\sin \left(\frac{\pi}{2}-\theta\right)\right) \\
\quad=\theta-\frac{\pi}{2} .
\end{aligned}
$$

In particular, when $\theta=\pi, A_{\text {hyp }}(T(A, B, \infty))=\pi / 2$. 
Lemma 5. Let $\gamma$ be a hyperbolic geodesic with two endpoints $A=c+t e^{i \theta_{1}}, B=c+t e^{i \theta_{2}}\left(c \in \mathbf{R}, t>0,0 \leq \theta_{1} \leq \pi, 0 \leq \theta_{2} \leq\right.$ $\left.\pi, \theta_{1} \neq \theta_{2}\right)$ in $\mathbf{H}^{2}$; then the hyperbolic area of the hyperbolic triangle $T(A, B, \infty)$ is as follows:

$$
A_{\text {hyp }}(T(A, B, \infty))=\left|\theta_{2}-\theta_{1}\right| \text {. }
$$

Proof. Let $C=c+i$. Without loss of generality, we assume $\theta_{1}<\theta_{2}$.

(1) If $0 \leq \theta_{1}<\theta_{2} \leq \pi / 2$, by Lemma 4 , we have

$$
\begin{aligned}
A_{\text {hyp }} & (T(A, B, \infty)) \\
& =A_{\text {hyp }}(T(A, C, \infty))-A_{\text {hyp }}(T(B, C, \infty)) \\
& =\left(\frac{\pi}{2}-\theta_{1}\right)-\left(\frac{\pi}{2}-\theta_{2}\right)=\theta_{2}-\theta_{1} .
\end{aligned}
$$

(2) If $\pi / 2 \leq \theta_{1}<\theta_{2} \leq \pi$, using Lemma 4 again, we get

$$
\begin{aligned}
A_{\text {hyp }} & (T(A, B, \infty)) \\
& =A_{\text {hyp }}(T(B, C, \infty))-A_{\text {hyp }}(T(A, C, \infty)) \\
& =\left(\theta_{2}-\frac{\pi}{2}\right)-\left(\theta_{1}-\frac{\pi}{2}\right)=\theta_{2}-\theta_{1} .
\end{aligned}
$$

(3) If $0 \leq \theta_{1}<\pi / 2<\theta_{2} \leq \pi$, Lemma 4 also implies

$$
\begin{aligned}
A_{\text {hyp }} & (T(A, B, \infty)) \\
& =A_{\text {hyp }}(T(A, C, \infty))+A_{\text {hyp }}(T(B, C, \infty)) \\
& =\left(\theta_{2}-\frac{\pi}{2}\right)+\left(\frac{\pi}{2}-\theta_{1}\right)=\theta_{2}-\theta_{1} .
\end{aligned}
$$

Similarly, if $\theta_{2}<\theta_{1}$ we have the same conclusion:

$$
A_{\text {hyp }}(T(A, B, \infty))=\theta_{1}-\theta_{2} \text {. }
$$

Thus, the proof of Lemma 5 is completed.

Theorem 2 is an application of Lemma 5. For the proof of Theorem 2 we also need a sharp formula of the circle orthogonal to the boundary of $\mathbf{H}^{2}$ [25].

Lemma A. Let $z_{1}, z_{2} \in \mathbf{H}^{2}$ with $x_{1} \neq x_{2}$. Then $S^{1}(c, r)$ is orthogonal to $\partial \mathbf{H}^{2}$, where

$$
c=\frac{\left|z_{1}\right|^{2}-\left|z_{2}\right|^{2}}{2\left(x_{1}-x_{2}\right)}, \quad r=\sqrt{y_{1}^{2}+\left(\frac{y_{2}^{2}+\left(x_{1}-x_{2}\right)^{2}-y_{1}^{2}}{2\left(x_{1}-x_{2}\right)}\right)^{2}} .
$$

Proof of Theorem 2. We may assume that $x_{1}>x_{2}$. Let $S^{1}(c, r)$ be the circle which is through the two points $z_{1}, z_{2}$, where $c$ and $r$ are represented as in (27). Then we have $z_{1}=c+r e^{i \theta_{1}}$, $z_{2}=c+r e^{i \theta_{2}}$.

Case $1\left(\left(x_{1}-c\right)\left(x_{2}-c\right)>0\right)$. If $c<x_{2}<x_{1}$, then by Lemma 5

$$
\begin{aligned}
A_{\text {hyp }} & \left(T\left(z_{1}, z_{2}, \infty\right)\right) \\
& =\left|\theta_{1}-\theta_{2}\right|=\arctan \frac{y_{2}}{x_{2}-c}-\arctan \frac{y_{1}}{x_{1}-c} .
\end{aligned}
$$

If $x_{2}<x_{1}<c$, so we have

$$
\begin{aligned}
A_{\text {hyp }}( & \left.T\left(z_{1}, z_{2}, \infty\right)\right) \\
& =\left|\theta_{1}-\theta_{2}\right|=\arctan \frac{y_{1}}{c-x_{1}}-\arctan \frac{y_{2}}{c-x_{2}} .
\end{aligned}
$$

Case $2\left(\left(x_{1}-c\right)\left(x_{2}-c\right)<0\right)$. This case implies that $x_{2}<c<x_{1}$; then

$$
\begin{aligned}
A_{\text {hyp }}( & \left.\left(z_{1}, z_{2}, \infty\right)\right) \\
& =\left|\theta_{1}-\theta_{2}\right|=\pi-\arctan \frac{y_{1}}{x_{1}-c}-\arctan \frac{y_{2}}{c-x_{2}} .
\end{aligned}
$$

Remark 6. For a general hyperbolic triangle $T(A, B, C)$ in $\mathbf{H}^{2}$, we can give its hyperbolic area formula by Theorem 2 . Given three points $A\left(x_{A}, y_{A}\right), B\left(x_{B}, y_{B}\right)$, and $C\left(x_{C}, y_{C}\right)$ in $\mathbf{H}^{2}$ with $\left(x_{A}-x_{B}\right)\left(x_{B}-x_{C}\right)\left(x_{C}-x_{A}\right) \neq 0$, we may assume that $x_{C}<x_{A}<x_{B}$. Let $S^{1}(c, r)$ be the circle which is through $B$ and $C$ and orthogonal to $\partial \mathbf{H}^{2}$. Let $D\left(x_{D}, y_{D}\right)$ be the point which is the intersection of $S^{1}(c, r)$ and the hyperbolic geodesic through point $A$ and orthogonal to $\partial \mathbf{H}^{2}$. Then we have

$$
x_{A}=x_{D}, \quad y_{D}=\sqrt{r^{2}-\left(x_{A}-c\right)^{2}}
$$

where $c=\left(\left|z_{B}\right|^{2}-\left|z_{C}\right|^{2}\right) / 2\left(x_{B}-x_{C}\right)$ and $r=$ $\sqrt{y_{B}^{2}+\left(\left(y_{C}^{2}+\left(x_{B}-x_{C}\right)^{2}-y_{B}^{2}\right) / 2\left(x_{B}-x_{C}\right)\right)^{2}}$.

Thus it follows

$$
\begin{aligned}
A_{\text {hyp }} & (T(A, B, C)) \\
& =A_{\text {hyp }}(T(A, C, D))+A_{\text {hyp }}(T(A, B, D)),
\end{aligned}
$$

where

$$
\begin{aligned}
A_{\text {hyp }}( & T(A, C, D)) \\
& =\left|A_{\text {hyp }}(T(A, C, \infty))-A_{\text {hyp }}(T(C, D, \infty))\right|, \\
A_{\text {hyp }}( & T(A, B, D)) \\
= & \left|A_{\text {hyp }}(T(A, B, \infty))-A_{\text {hyp }}(T(B, D, \infty))\right| ;
\end{aligned}
$$

then by Theorem 2 we can get the result of $A_{\text {hyp }}(T(A, B, C))$.

Example 7. Let $A=i, B=1+2 i, C=-1+3 i$; by calculation we have

$$
\begin{aligned}
A_{\text {hyp }} & (T(A, B, C)) \\
& =A_{\text {hyp }}(T(A, C, D))+A_{\text {hyp }}(T(A, B, D)) \approx 0.372,
\end{aligned}
$$

where $D=\sqrt{30} i / 2$.

Remark 8. There are lots of triangles which have the same hyperbolic area but different Euclidean areas in $\mathbf{H}^{2}$. For 
instance, $T(A, B, C)$ and $T(D, B, E)$ are two hyperbolic triangles in $\mathbf{H}^{2}$ with $A=-1, B=0, C=1, D=-2, E=2$, and

$$
\begin{gathered}
A_{\text {hyp }}(T(A, B, C))=A_{\text {hyp }}(T(D, B, E))=\pi, \\
\frac{\pi}{4}=A_{\text {euc }}(T(A, B, C)) \neq A_{\text {euc }}(T(D, B, E))=\pi .
\end{gathered}
$$

Remark 9. For any hyperbolic $n$-polygons $P\left(A_{1}, A_{2}, \ldots, A_{n}\right)$ $(n \geq 3)$ in $\mathbf{H}^{2}$, we always can divide it into several hyperbolic triangles $T_{1}, T_{2}, \ldots, T_{i}(i \geq 1)$; then we have

$$
A_{\text {hyp }}\left(P\left(A_{1}, A_{2}, \ldots, A_{n}\right)\right)=\sum_{k=1}^{i} A_{\text {hyp }}\left(T_{k}\right) \text {. }
$$

Remark 10. For any hyperbolic n-polygons $P\left(A_{1}, A_{2}, \ldots, A_{n}\right)$ $(n \geq 3)$ in $\mathbf{B}^{2}$, since the hyperbolic area is invariant under Möbius transformation, so we can map the $P\left(A_{1}, A_{2}, \ldots, A_{n}\right)$ into $\mathbf{H}^{2}$ by a Möbius transformation; therefore we obtain the hyperbolic area of $P\left(A_{1}, A_{2}, \ldots, A_{n}\right)$ in $\mathbf{B}^{2}$.

\section{Hyperbolic Areas of Lambert Quadrilaterals}

As an application of the explicit hyperbolic area formula of a hyperbolic triangle, we obtain the hyperbolic area of a Lambert quadrilateral.

Theorem 11. Let $Q(A, B, C, D)$ be the Lambert quadrilateral with angles $(\pi / 2, \pi / 2, \phi, \pi / 2), 0 \leq \phi<\pi / 2$ in $\mathbf{H}^{2}$ and $A=$ $c_{0}+i r_{0}, B=c_{0}+i r ;$ then

(1) if $r \leq d$,

$$
\begin{aligned}
A_{\text {hyp }} & (Q(A, B, C, D)) \\
& =\arctan \frac{y_{2}}{c_{1}-x_{2}}+\arctan \frac{y_{2}}{x_{2}-c_{0}}-\frac{\pi}{2},
\end{aligned}
$$

(2) if $d<r<c_{1}+r_{1}-c_{0}$,

$$
\begin{aligned}
A_{\text {hyp }} & (Q(A, B, C, D)) \\
& =\frac{\pi}{2}+\arctan \frac{y_{2}}{x_{2}-c_{0}}-\arctan \frac{y_{2}}{c_{1}-x_{2}},
\end{aligned}
$$

where $d=\sqrt{\left(c_{1}-c_{0}\right)^{2}+r_{1}^{2}}, C=x_{2}+i y_{2}, D=x_{1}+i y_{1}, c_{1}$ and $r_{1}$ are given at (39).

Proof. Without loss of generality, we assume that $c_{0}<c_{1}, 0<$ $r_{0}<r$ and $C \in S^{1}\left(c_{0}, r\right) \cap S^{1}\left(c_{1}, r_{1}\right), D \in S^{1}\left(c_{0}, r_{0}\right) \cap S^{1}\left(c_{1}, r_{1}\right)$ (see Figure 1), which implies $c_{0}+r_{0}>c_{1}-r_{1}, c_{1}+r_{1}>c_{0}+r$. By (27) we have

$$
\begin{gathered}
c_{1}=\frac{x_{1}^{2}+y_{1}^{2}-x_{2}^{2}-y_{2}^{2}}{2\left(x_{1}-x_{2}\right)}, \\
r_{1}=\sqrt{y_{1}^{2}+\left(\frac{y_{2}^{2}+\left(x_{1}-x_{2}\right)^{2}-y_{1}^{2}}{2\left(x_{1}-x_{2}\right)}\right)^{2}} .
\end{gathered}
$$

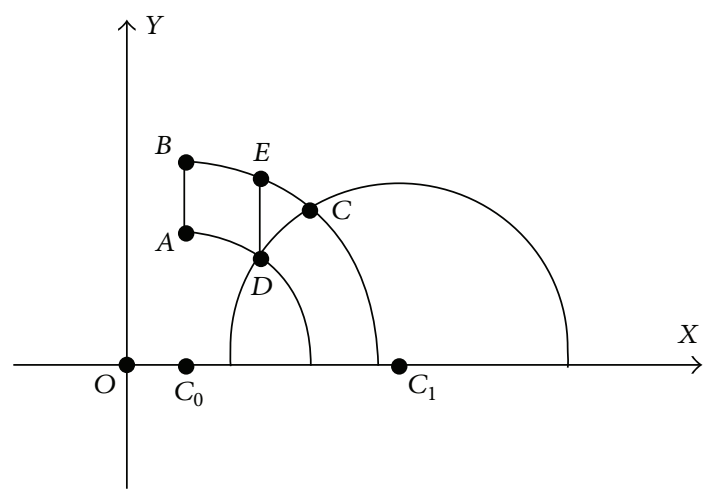

Figure 1

When $r \leq d$, we get

$$
\begin{aligned}
A_{\text {hyp }} & (Q(A, B, C, D)) \\
& =A_{\text {hyp }}(Q(A, B, E, D))+A_{\text {hyp }}(T(E, C, D)),
\end{aligned}
$$

where $E=x_{1}+i \sqrt{r^{2}-\left(x_{1}-c_{0}\right)^{2}}$. So, the following three relations

$$
\begin{aligned}
& A_{\text {hyp }}(Q(A, B, E, D)) \\
&=A_{\text {hyp }}(T(A, D, \infty))-A_{\text {hyp }}(T(B, E, \infty)), \\
& A_{\text {hyp }}(T(E, C, D)) \\
& \quad= A_{\text {hyp }}(T(D, C, \infty))-A_{\text {hyp }}(T(E, C, \infty)), \\
&\left|c_{1}-c_{0}\right|^{2}=\left(x_{1}-c_{0}\right)^{2}+y_{1}^{2}+\left(x_{1}-c_{1}\right)^{2}+y_{1}^{2},
\end{aligned}
$$

imply that

$$
A_{\text {hyp }}(Q(A, B, C, D))=\arctan \frac{y_{2}}{c_{1}-x_{2}}+\arctan \frac{y_{2}}{x_{2}-c_{0}}-\frac{\pi}{2} .
$$

When $d<r<c_{1}+r_{1}-c_{0}$, it follows

$$
\begin{aligned}
& A_{\text {hyp }}(Q(A, B, C, D)) \\
& =A_{\text {hyp }}(Q(A, B, E, D))+A_{\text {hyp }}(T(E, C, D)) \text {, } \\
& A_{\text {hyp }}(Q(A, B, E, D)) \\
& =A_{\text {hyp }}(T(A, D, \infty))-A_{\text {hyp }}(T(B, E, \infty)), \\
& A_{\text {hyp }}(T(E, C, D)) \\
& =A_{\text {hyp }}(T(D, C, \infty))-A_{\text {hyp }}(T(E, C, \infty)),
\end{aligned}
$$

and then we get

$$
\begin{aligned}
A_{\text {hyp }} & (Q(A, B, C, D)) \\
& =\frac{\pi}{2}+\arctan \frac{y_{2}}{x_{2}-c_{0}}-\arctan \frac{y_{2}}{x_{2}-c_{1}} .
\end{aligned}
$$

The proof of the case that $c_{0}>c_{1}$ is similar to the case that $c_{1}>c_{0}$. So the proof of Theorem 11 is complete. 


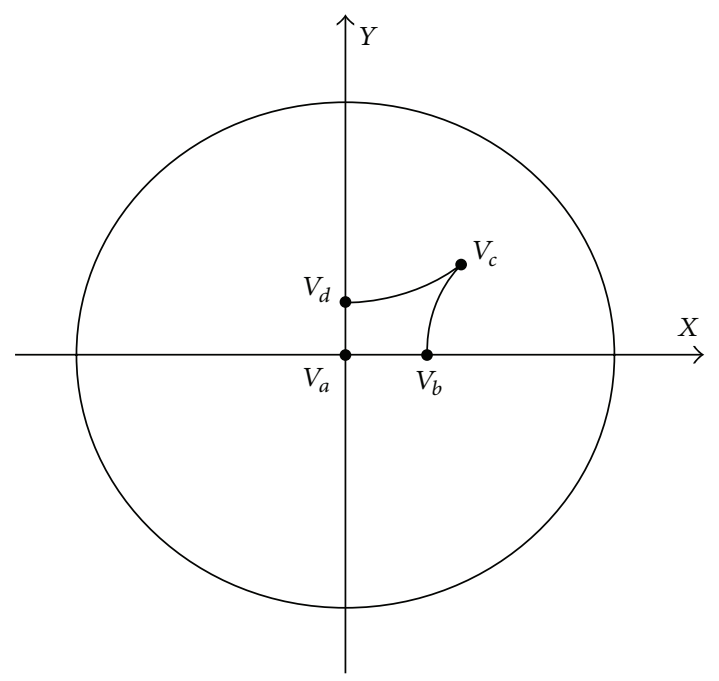

Figure 2

In particular, when $r=c_{1}+r_{1}-c_{0}$, we have

$$
A_{\text {hyp }}(Q(A, B, C, D))=\frac{\pi}{2} \text {. }
$$

Remark 12. Since the Saccheri quadrilateral can be divided into two Lambert quadrilaterals, therefore the hyperbolic area of a Saccheri quadrilateral is twice of a Lambert quadrilateral.

\section{Hyperbolic Geometric Characterization of Lambert Quadrilaterals and Saccheri Quadrilaterals}

Lemma $\mathbf{B}$ (see [25]). Let $a \in \mathbf{R}^{2}$ be a constant with $|a|>1$. Then $S^{1}(a, r)$ is orthogonal to $S^{1}(0,1)$ for $|a|^{2}=1+r^{2}$. Given $z_{1}=x_{1}+i y_{1}, z_{2}=x_{2}+i y_{2} \in \mathbf{B}^{2}$ such that $0, z_{1}$, and $z_{2}$ are noncollinear, the orthogonal circle $S^{1}(a, r)$ contains $z_{1}$ and $z_{2}$ if

$$
\begin{gathered}
a=i \frac{z_{2}\left(1+\left|z_{1}\right|^{2}\right)-z_{1}\left(1+\left|z_{2}\right|^{2}\right)}{2\left(x_{2} y_{1}-x_{1} y_{2}\right)}, \\
r=\frac{\left.\left|z_{1}-z_{2}\right|\left|z_{1}\right| z_{2}\right|^{2}-z_{2} \mid}{2\left|z_{2}\right|\left|x_{1} y_{2}-x_{2} y_{1}\right|}
\end{gathered}
$$

and $S^{1}(a, r) \cap S^{1}(0,1)=\left\{z \in \mathbf{R}^{2}: z=a /|a| \exp ( \pm i \theta), \theta=\right.$ $\arccos (1 /|\mathrm{a}|)\}$.

The following result in Lemma 13 appeared in [20]; for completeness, we give its proof as follows.

Lemma 13. Let $\mathrm{Q}\left(v_{a}, v_{b}, v_{c}, v_{d}\right)$ be a Lambert quadrilateral (see Figure 2) in $\mathbf{B}^{2}$ with $v_{c}=t e^{i \theta}, 0<\theta<\pi / 2$ and let

$$
\begin{aligned}
& d_{1}=d_{\rho}\left(J\left[v_{a}, v_{d}\right], J\left[v_{b}, v_{c}\right]\right), \\
& d_{2}=d_{\rho}\left(J\left[v_{a}, v_{b}\right], J\left[v_{c}, v_{d}\right]\right) ;
\end{aligned}
$$

then

$$
d_{1}=\operatorname{arth}(L r), \quad d_{2}=\operatorname{arth}\left(L r^{\prime}\right),
$$

where $L=\tanh \rho_{\mathbf{B}^{2}}\left(v_{a}, v_{c}\right), r=\cos \theta, r^{\prime}=\sqrt{1-r^{2}}=\sin \theta$.

Proof. Assume that $v_{a}=0, v_{b}$ is on the real axis, $v_{d}$ is on the imaginary axis, and $v_{c}=t e^{i \theta}, 0<t \leq 1$ and $0<\theta<\pi / 2$. We use (10) to obtain

$$
\begin{gathered}
\rho_{\mathbf{B}^{2}}\left(0, v_{c}\right)=\log \frac{1+\left|v_{c}\right|}{1-\left|v_{c}\right|}=\log \frac{1+t}{1-t}, \\
L=\tanh \rho_{\mathbf{B}^{2}}\left(v_{a}, v_{c}\right)=\frac{2 t}{1+t^{2}} .
\end{gathered}
$$

Utilizing the relation (46), we have

$$
\begin{array}{ll}
d=i \frac{1+t^{2}}{2 t \sin \theta}, & r_{d}=\frac{\sqrt{\left(1+t^{2}\right)^{2}-4 t^{2} \sin ^{2} \theta}}{2 t \sin \theta}, \\
b=\frac{1+t^{2}}{2 t \cos \theta}, & r_{b}=\frac{\sqrt{\left(1+t^{2}\right)^{2}-4 t^{2} \cos ^{2} \theta}}{2 t \cos \theta}
\end{array}
$$

where $b, r_{b}$ denote the center and radius of the circle through the geodesic $\gamma\left(v_{b}, v_{c}\right)$ and $d$ and $r_{d}$ denote the center and radius of the circle through the geodesic $\gamma\left(v_{c}, v_{d}\right)$. Write $P(b+$ $\left.r_{b} \cos \psi, r_{b} \sin \psi\right) \in \gamma\left(v_{b}, v_{c}\right), \pi / 2<\psi<\pi$ and $Q(0, y) \epsilon$ $\gamma\left(v_{a}, v_{d}\right), 0 \leq y \leq\left|v_{d}\right|=|d|-r_{d}$. Let $f(\psi, y)=\rho_{\mathbf{B}^{2}}(P, Q)$. Then the relation (9) implies that

$$
\begin{aligned}
f(\psi, y) & =\rho_{\mathrm{B}^{2}}(P, Q) \\
& =2 \operatorname{arth} \frac{\left|z_{P}-z_{\mathrm{Q}}\right|}{\sqrt{\left|z_{P}-z_{\mathrm{Q}}\right|^{2}+\left(1-\left|z_{P}\right|^{2}\right)\left(1-\left|z_{\mathrm{Q}}\right|^{2}\right)}} \\
& =2 \operatorname{arth} \sqrt{\frac{b^{2}+r_{b}^{2}+2 b r_{b} \cos \psi-2 y r_{b} \sin \psi+y^{2}}{1+y^{2}\left(b^{2}+r_{b}^{2}+2 b r_{b} \cos \psi\right)-2 y r_{b} \sin \psi}} .
\end{aligned}
$$

From the relation (7) we know that $d_{1}=\inf _{\psi, y} \rho_{\mathbf{B}^{2}}(P, Q)$. The function $f(\psi, y)$ reaches the minimum value

$$
f(\pi, 0)=2 \operatorname{arth}\left(b-r_{b}\right)=\operatorname{arth} \frac{2 t \cos \theta}{1+t^{2}} .
$$

Then

$$
d_{1}=\rho_{\mathbf{B}^{2}}\left(0, v_{b}\right)=\operatorname{arth} \frac{2 t \cos \theta}{1+t^{2}}=\operatorname{arth}(L r),
$$

where $r=\cos \theta$. Similarly, we have

$$
d_{2}=\rho_{\mathrm{B}^{2}}\left(0, v_{d}\right)=\operatorname{arth} \frac{2 t \sin \theta}{1+t^{2}}=\operatorname{arth}\left(L r^{\prime}\right) \text {, }
$$

where $r^{\prime}=\sqrt{1-r^{2}}=\sin \theta$. 
Proof of Theorem 3. Assume that $v_{a}=0$ and $v_{c}=t e^{i \theta}, 0<t \leq$ $1,0<\theta<\pi / 2$ and let $v_{b}$ be on the real axis and $v_{d}$ on the imaginary axis (see Figure 2). We use (10) to obtain

$$
\begin{gathered}
\rho_{\mathrm{B}^{2}}\left(0, v_{c}\right)=\log \frac{1+\left|v_{c}\right|}{1-\left|v_{c}\right|}=\log \frac{1+t}{1-t}, \\
L=\tanh \rho_{\mathrm{B}^{2}}\left(v_{a}, v_{c}\right)=\frac{2 t}{1+t^{2}},
\end{gathered}
$$

which implies

$$
\rho_{\mathbf{B}^{2}}\left(v_{a}, v_{c}\right)=\operatorname{arth} \frac{2 t}{1+t^{2}} .
$$

By the relation (46) we have that the geodesic $\gamma\left(v_{b}, v_{c}\right)$ is on the circle $S^{1}\left(b, r_{b}\right)$, where

$$
r_{b}=\frac{\sqrt{\left(1+t^{2}\right)^{2}-4 t^{2} \cos ^{2} \theta}}{2 t \cos \theta}, \quad b=\frac{1+t^{2}}{2 t \cos \theta} .
$$

Similarly, the geodesic $\gamma\left(v_{c}, v_{d}\right)$ is on the circle $S^{1}\left(d, r_{d}\right)$, where

$$
d=i \frac{1+t^{2}}{2 t \sin \theta}, \quad r_{d}=\frac{\sqrt{\left(1+t^{2}\right)^{2}-4 t^{2} \sin ^{2} \theta}}{2 t \sin \theta} .
$$

Hence, $t_{1}=\left|v_{b}-v_{a}\right|=b-r_{b}$ and $t_{2}=\left|v_{d}-v_{a}\right|=|d|-r_{d}$. Utilizing the relation (9), we obtain

$$
\begin{aligned}
\rho_{\mathrm{B}^{2}}\left(v_{b}, v_{d}\right) & =2 \operatorname{arth} \frac{\left|v_{b}-v_{d}\right|}{\sqrt{\left|v_{b}-v_{d}\right|^{2}+\left(1-\left|v_{b}\right|^{2}\right)\left(1-\left|v_{d}\right|^{2}\right)}} \\
& =2 \operatorname{arth} \sqrt{\frac{t_{1}^{2}+t_{2}^{2}}{1+t_{1}^{2} t_{2}^{2}}} .
\end{aligned}
$$

This completes the proof of Theorem 3.

Theorem 14. Let $S\left(v_{d}^{\prime}, v_{d}, v_{c}, v_{c}^{\prime}\right)$ be a Saccheri quadrilateral in $\mathbf{B}^{2}$ with $v_{c}=t e^{i \theta}, v_{c}^{\prime}=t e^{-i \theta}, 0<\theta<\pi / 2$ and

$$
\begin{aligned}
& d_{3}=d_{\rho}\left(J\left[v_{c}, v_{c}^{\prime}\right], J\left[v_{d}, v_{d}^{\prime}\right]\right), \\
& d_{4}=d_{\rho}\left(J\left[v_{c}, v_{d}\right], J\left[v_{c}^{\prime}, v_{d}^{\prime}\right]\right) ;
\end{aligned}
$$

then

$$
\tanh ^{2} d_{3}+\tanh ^{2}\left(\frac{d_{4}}{2}\right)=L^{2},
$$

where $L=\tanh \rho_{\mathbf{B}^{2}}\left(0, v_{c}\right)$.

Proof. Assume that $v_{c}=t e^{i \theta}, 0<t \leq 1,0<\theta<\pi / 2$ and $v_{a}=0$ and let $v_{b}$ be on the real axis and $v_{d}$ on the imaginary axis (see Figure 3). Then by the relation (46) we have that the circle through points $v_{c}$ and $v_{c}^{\prime}$ which is orthogonal to $\mathbf{B}^{2}$ is $S^{1}\left(b, r_{b}\right)$, where

$$
b=\frac{1+t^{2}}{2 t \cos \theta}, \quad r_{b}=\frac{\sqrt{\left(1+t^{2}\right)^{2}-4 t^{2} \cos ^{2} \theta}}{2 t \cos \theta} .
$$

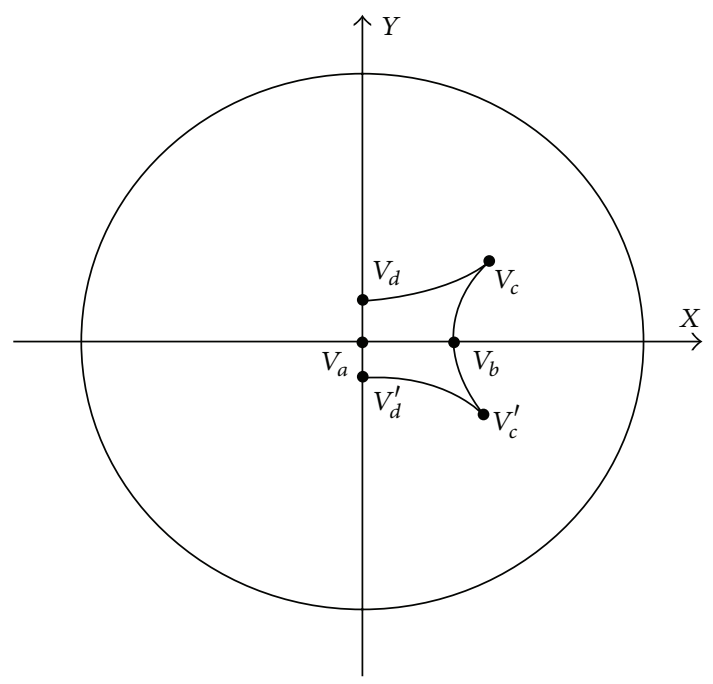

Figure 3

It follows from the relations (10) and (48) that

$$
\begin{aligned}
& d_{3}=\rho_{\mathbf{B}^{2}}\left(0, v_{b}\right)=\operatorname{arth}(L \cos \theta), \\
& d_{4}=2 \rho_{\mathbf{B}^{2}}\left(0, v_{d}\right)=2 \operatorname{arth}(L \sin \theta),
\end{aligned}
$$

where $L=\tanh \rho_{\mathbf{B}^{2}}\left(0, v_{c}\right)=2 t /\left(1+t^{2}\right) \in(0,1]$. Thus,

$$
\tanh ^{2} d_{3}+\tanh ^{2}\left(\frac{d_{4}}{2}\right)=L^{2} .
$$

This completes the proof of Theorem 14.

\section{Conflict of Interests}

The authors declare that there is no conflict of interests regarding the publication of this paper.

\section{Acknowledgments}

This paper is supported by the Natural Science Foundation of Fujian Province of China (2014J01013), NCETFJ Fund (2012FJ-NCET-ZR05), and the Promotion Program for Young and Middle-Aged Teacher in Science and Technology Research of Huaqiao University (ZQN-YX110).

\section{References}

[1] A. F. Beardon and C. Pommerenke, "The Poincaré metric of plane domains," The Journal of the London Mathematical Society II, vol. 18, no. 3, pp. 475-483, 1978.

[2] B. Iversen, Hyperbolic Geometry, vol. 25 of London Mathematical Society Student Texts, Cambridge University Press, Cambridge, UK, 1992.

[3] J. Milnor, "Hyperbolic geometry: the first 150 years," American Mathematical Society, vol. 6, no. 1, pp. 9-24, 1982.

[4] S. Kulczycki, Non-Euclidean Geometry, Courier Dover, Mineola, NY, USA, 2012. 
[5] A. F. Beardon, The Geometry of Discrete Groups, vol. 91 of Graduate Texts in Mathematics, Springer, New York, NY, USA, 1983.

[6] M. Vuorinen, Conformal Geometry and Quasiregular Mappings, vol. 1319 of Lecture Notes in Mathematics, Springer, Berlin, Germany, 1988.

[7] J. W. Anderson, Hyperbolic Geometry, Springer Undergraduate Mathematics Series, Springer, London, UK, 2nd edition, 2005.

[8] L. Keen and N. Lakic, Hyperbolic Geometry from a Local Viewpoint, London Mathematical Society Student Texts 68, Cambridge University Press, Cambridge, UK, 2007.

[9] A. Marden, Outer Circles: An introduction to hyperbolic 3manifolds, Cambridge University Press, Cambridge, UK, 2007.

[10] J. G. Ratcliffe, Foundations of Hyperbolic Manifolds, Graduate Texts in Mathmatics, Springer, New York, NY, USA, 2nd edition, 2006.

[11] N. Curien and W. Werner, "The Markovian hyperbolic triangulation," Journal of the European Mathematical Society, vol. 15, no. 4, pp. 1309-1341, 2013.

[12] O. Demirel, "A characterization of Möbius transformations by use of hyperbolic triangles," Journal of Mathematical Analysis and Applications, vol. 398, no. 2, pp. 457-461, 2013.

[13] A. N. Fang and S. H. Yang, "Corrigendum to 'a new characteristic of Möbius transformations in hyperbolic geometry' [Journal of Mathematical Analysis and Applications, vol. 319, no. 2, pp. 660-664, 2006]," Journal of Mathematical Analysis and Applications, vol. 376, pp. 383-384, 2011.

[14] S. Yang and A. Fang, "A new characteristic of Möbius transformations in hyperbolic geometry," Journal of Mathematical Analysis and Applications, vol. 319, no. 2, pp. 660-664, 2006.

[15] V. Pambuccian, "Mappings preserving the area equality of hyperbolic triangles are motions," Archiv der Mathematik, vol. 95, no. 3, pp. 293-300, 2010.

[16] X. Chen and A. Fang, "A class of harmonic quasiconformal mappings with strongly hyperbolically convex ranges," Complex Variables and Elliptic Equations, vol. 58, no. 7, pp. 1013-1022, 2013.

[17] X. D. Chen and A. N. Fang, "A Schwarz-Pick inequality for harmonic quasiconformal mappings and its applications," Journal of Mathematical Analysis and Applications, vol. 369, no. 1, pp. 22-28, 2010.

[18] M. Knežević and M. Mateljević, "On the quasi-isometries of harmonic quasiconformal mappings," Journal of Mathematical Analysis and Applications, vol. 334, no. 1, pp. 404-413, 2007.

[19] M. Rostamzadeh and S. Taherian, "Defect and area in BeltramiKlein model of hyperbolic geometry," Results in Mathematics, vol. 63, no. 1-2, pp. 229-239, 2013.

[20] M. Vuorinen and G. Wang, "Hyperbolic Lambert quadrilaterals and quasiconformal mappings," Annales Academice Scientiarum Fennicee Mathematica, vol. 38, no. 2, pp. 433-453, 2013.

[21] N. Kanesaka and H. Nakamura, "On hyperbolic area of the moduli of $\theta$-acute triangles," Mathematical Journal of Okayama University, vol. 55, pp. 191-200, 2013.

[22] F. W. Gehring and E. Reich, "Area distortion under quasiconformal mappings," Annales Academiae Scientiarum Fennicae A, vol. 388, pp. 1-15, 1966.

[23] J. A. Kelingos, "Distortion of hyperbolic area under quasiconformal mappings," Duke Mathematical Journal, vol. 41, pp. 127139, 1974.

[24] R. M. Porter and L. F. Reséndis, "Quasiconformally explodable sets," Complex Variables: Theory and Application, vol. 36, no. 4, pp. 379-392, 1998.
[25] R. Klén and M. Vuorinen, "Apollonian circles and hyperbolic geometry," Journal of Analysis, vol. 19, pp. 41-60, 2011. 


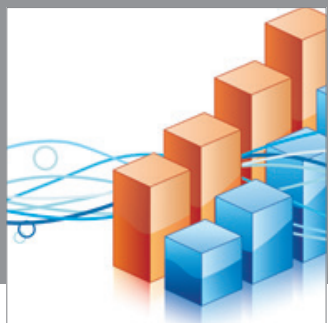

Advances in

Operations Research

mansans

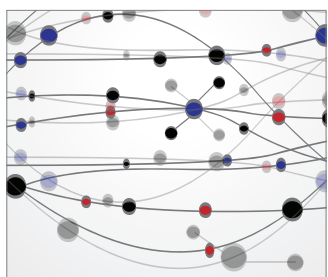

The Scientific World Journal
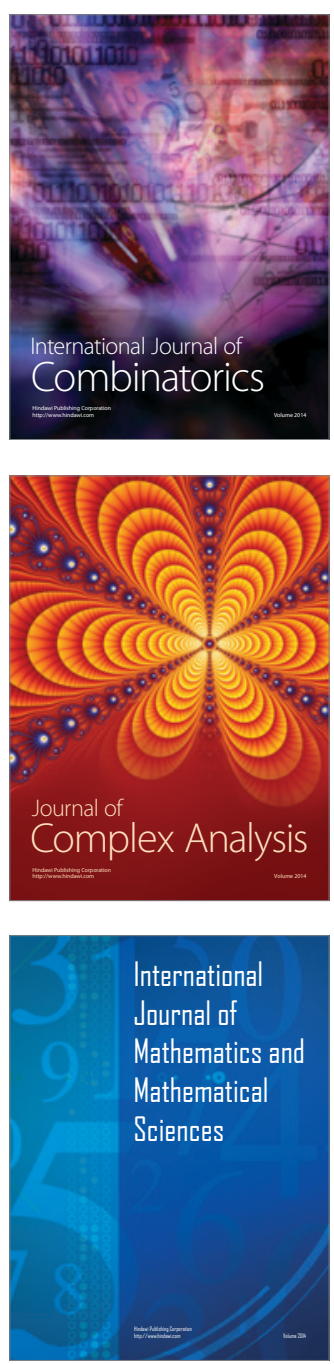
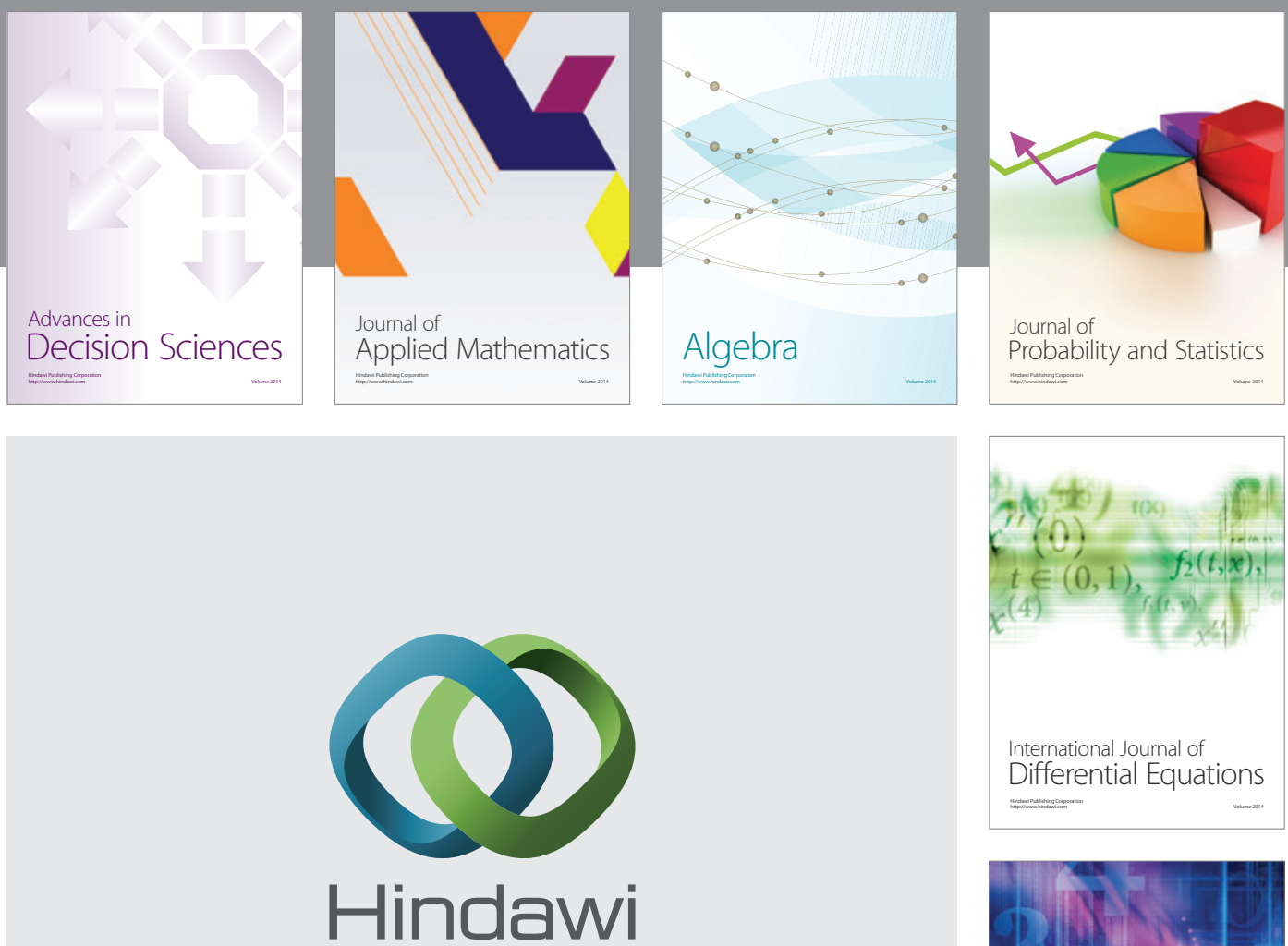

Submit your manuscripts at http://www.hindawi.com
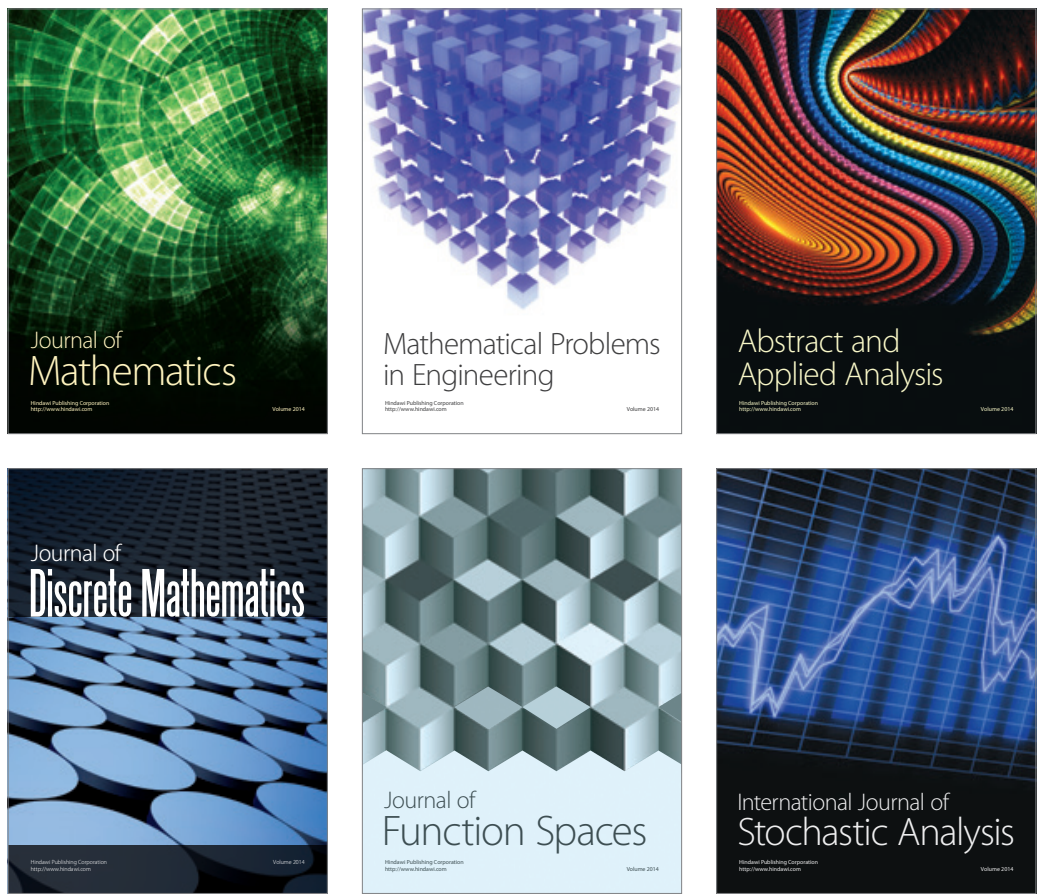

Journal of

Function Spaces

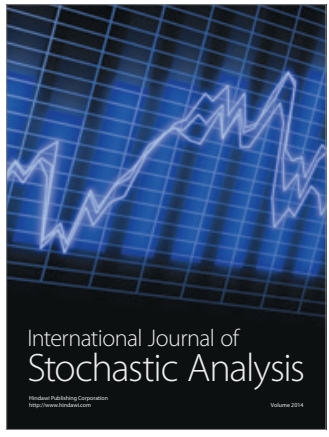

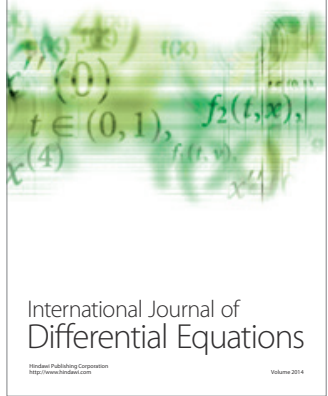
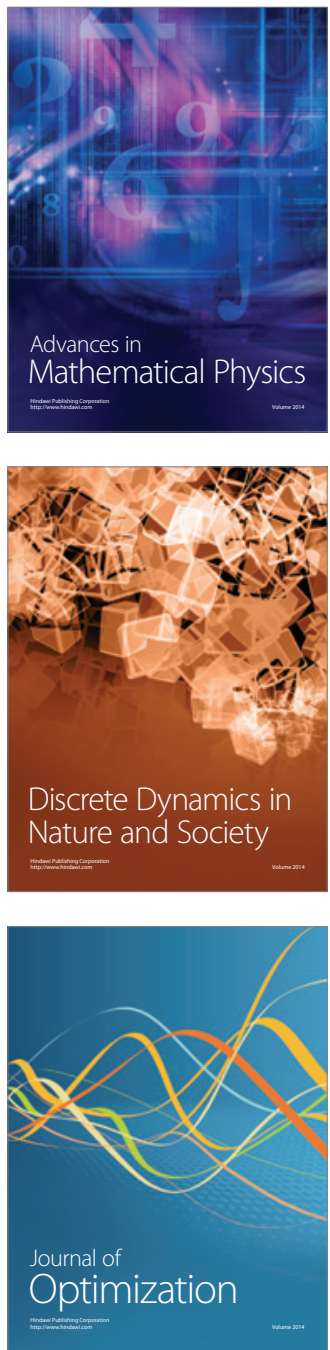